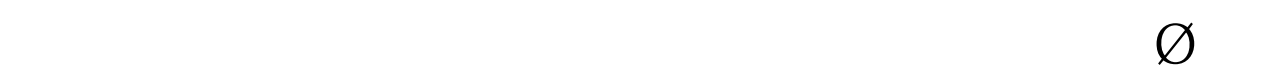 rftéciir
}

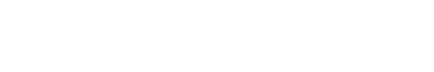

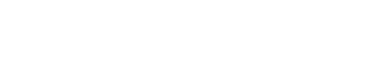

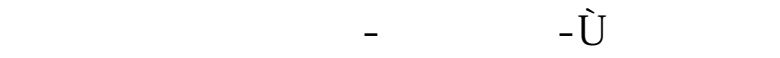

ŭ Ťsin

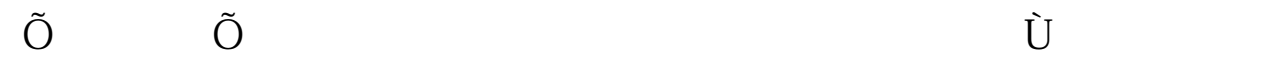

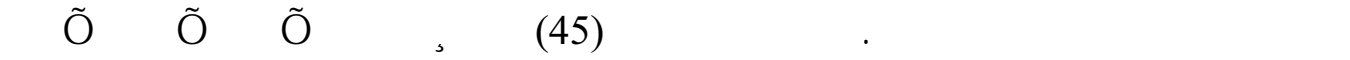

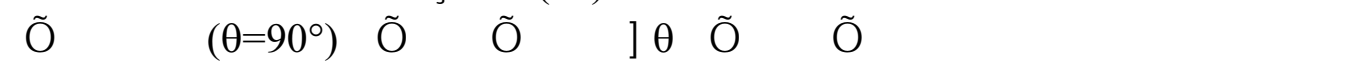

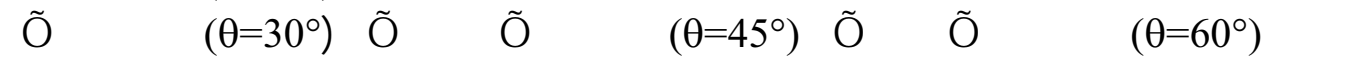

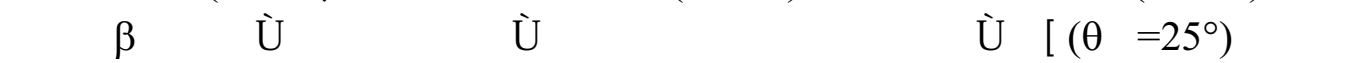

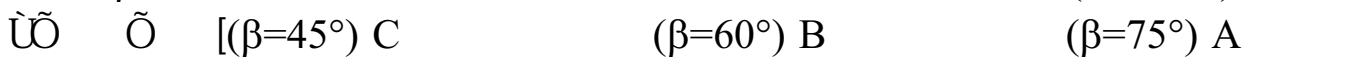

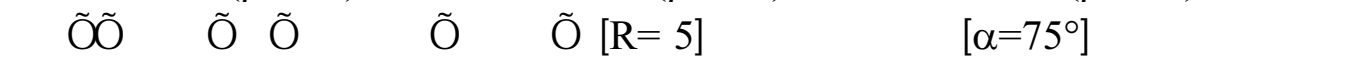

$$
\text { . } \mathrm{C}^{\mathrm{U}}[\mathrm{P}=30,20,10]
$$

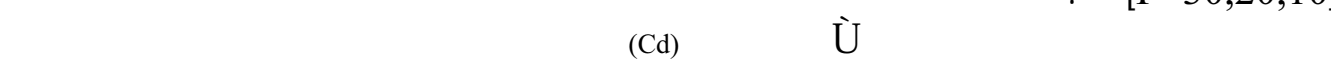

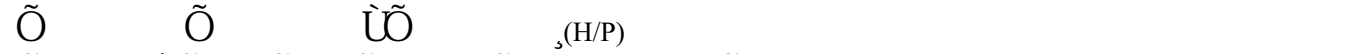

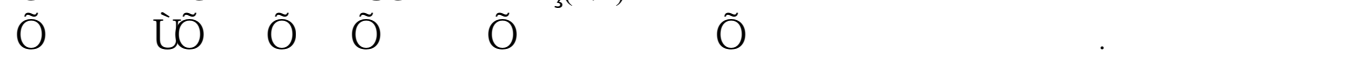

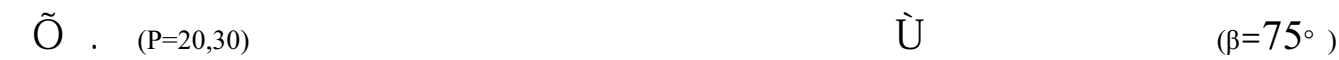

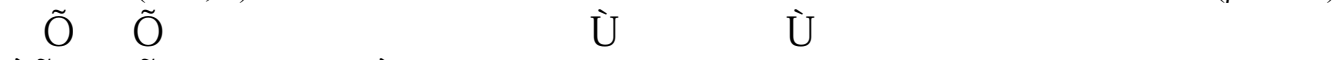

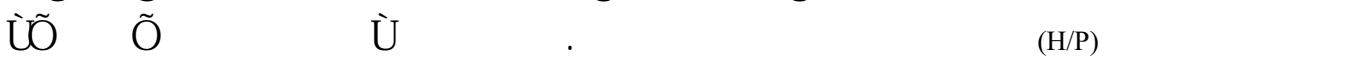

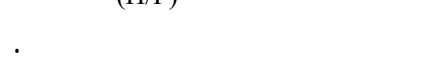

\section{Coefficient of Discharge For the Normal and Oblique Weirs with Semi-Circular Crest}

\author{
H.A.HAYAWI S. J. AL-MOULA
}

University of Mosul - College of Eng. -Water Resource Eng. Dept.

\section{ABSTRACT}

The coefficient of discharge of normal and oblique weirs with semicircular crest under free over flow conditions was studied experimentally. Forty-five weir models were constructed and tested.These models classified in to five groups based on the variation of oblique angle with the canal wall $(\theta)$ [group one $\left(\theta=90^{\circ}\right)$, group two $\left(\theta=60^{\circ}\right)$, group three $\left(\theta=45^{\circ}\right)$, group four $\left(\theta=30^{\circ}\right)$, group five $\left.\left(\theta=25^{\circ}\right)\right]$. Each group included three series of tests depending on the variation of downstream face slope $(\beta)$ [series A $\left(\beta=75^{\circ}\right)$, series $B\left(\beta=60^{\circ}\right)$, series $\mathrm{C}$ $\left.\left(\beta=45^{\circ}\right)\right]$.The upstream slop was fixed for all models with angle $\left(\alpha=75^{\circ}\right)$ and the crest radius $(\mathrm{R}=5) \mathrm{cm}$. Every series included testing of three models based on the variation of weir height $[\mathrm{P}=30,20 \& 10 \mathrm{~cm}]$.

The experimental results of the study showed that the discharge coefficient (Cd) increases with the increase of water depth above the crest to the weir height ratio $(H / P)$, and with the increase of downstream slope $(\beta)$ the peak values of $(\mathrm{Cd})$ were found when downstream face slope $\left(\beta=75^{\circ}\right)$ and weir height $(\mathrm{P}=10 \mathrm{~cm})$. In case of oblique weirs, it was found that $(\mathrm{Cd})$ decreases with increase of $(\mathrm{H} / \mathrm{P})$ values. Also it was found that $(\mathrm{Cd})$ increases with the increase of the downstream slope $(\beta) \&$ weirs of small oblique angle $(\theta)$ give low values of $(\mathrm{Cd})$.

Keywords: Weirs; Free-fall Structures Experimental Study 
\begin{tabular}{llll} 
Al-Rafidain Engineering & Vol.14 & No.2 & 2006 \\
\hline
\end{tabular}

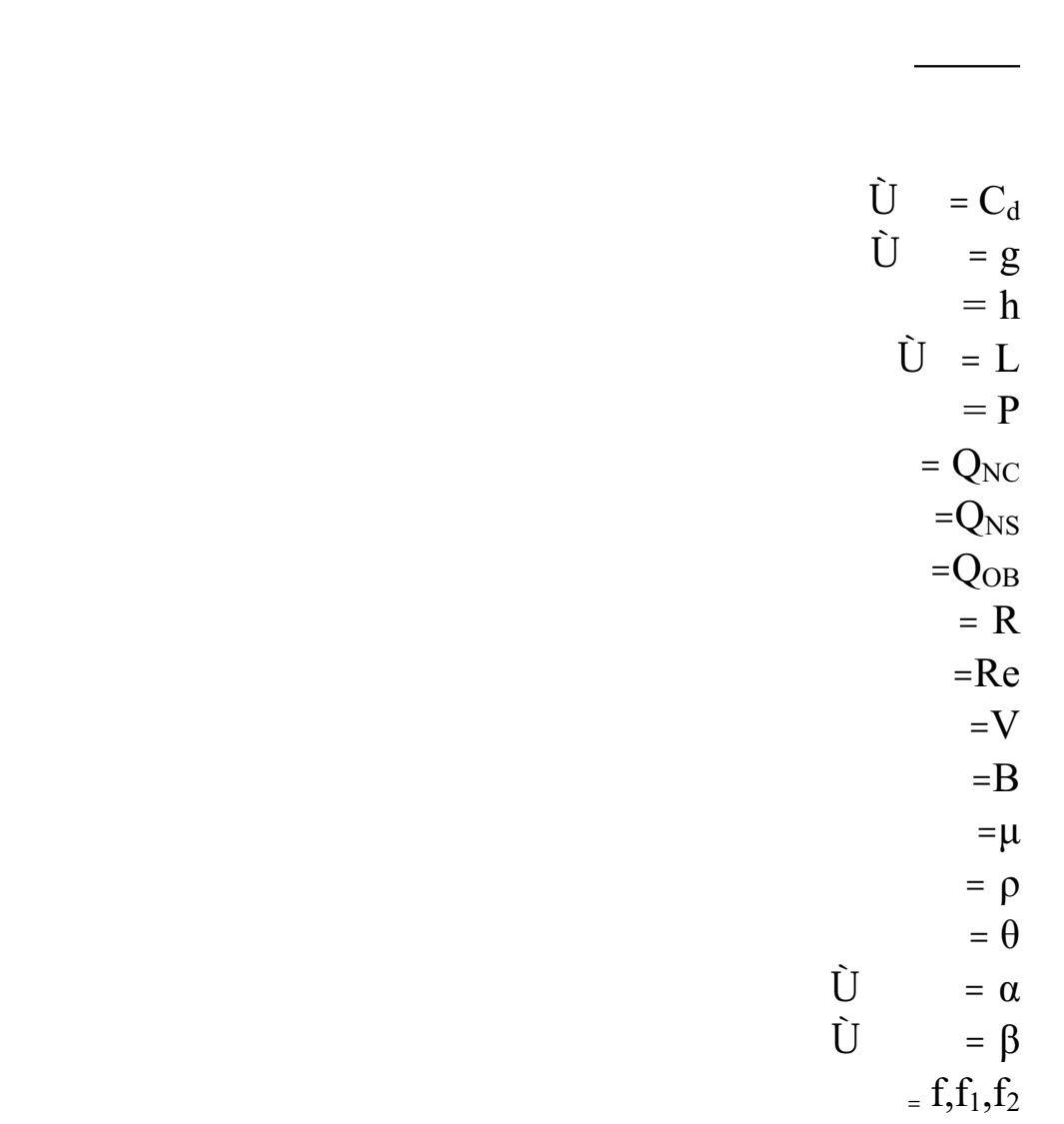

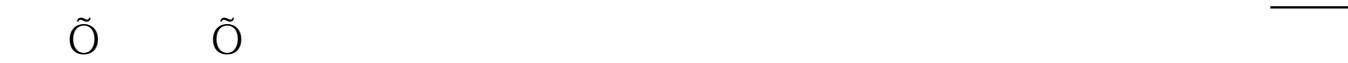

retsin

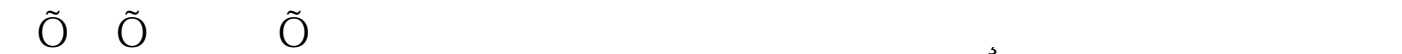

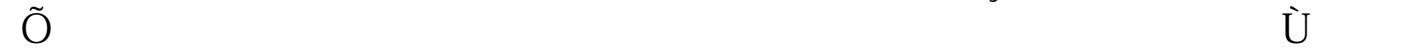

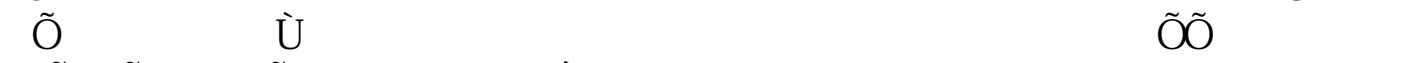

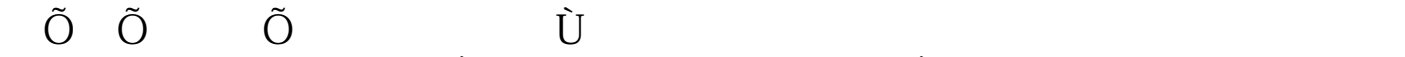

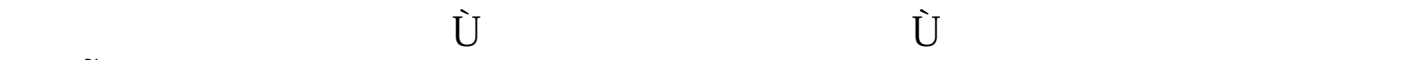

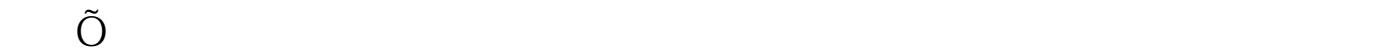

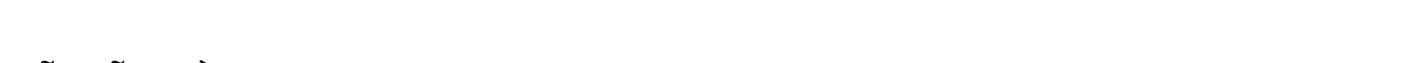

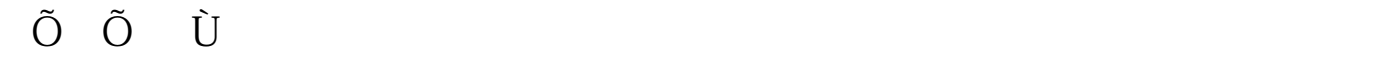

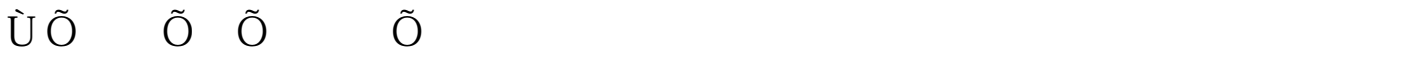

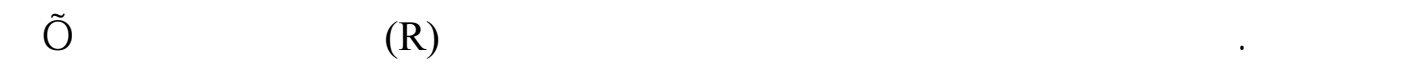

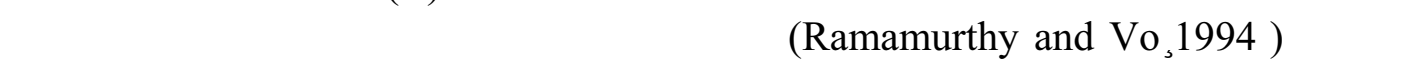

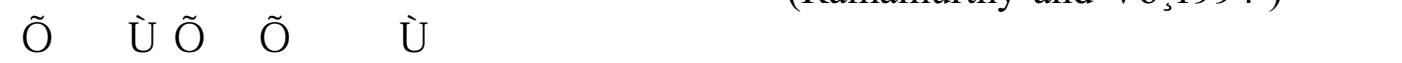
.ř

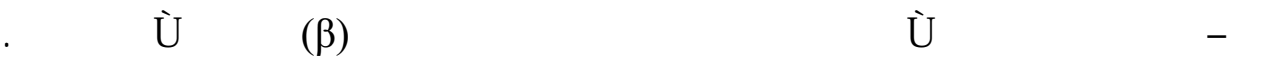

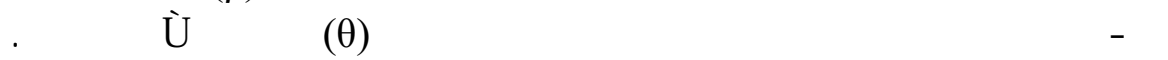
¿đ̛̛̛̃CF

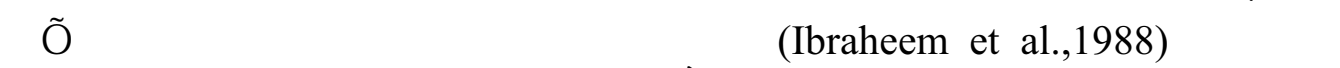
.Ž ชü ș́

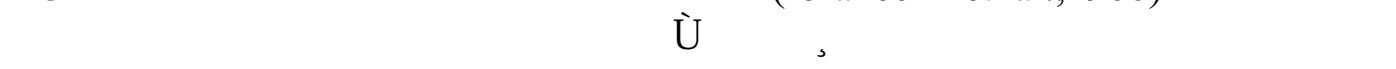




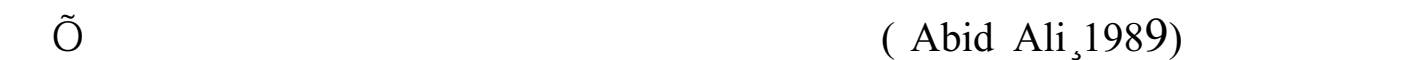

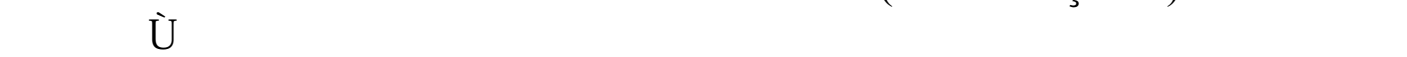

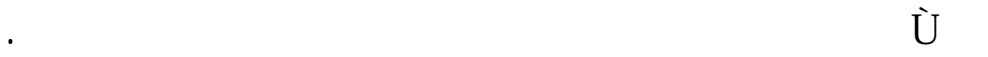

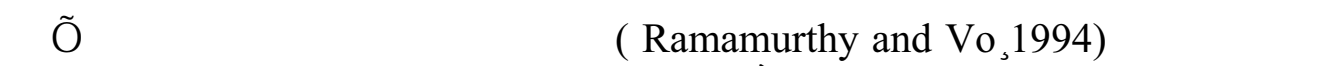

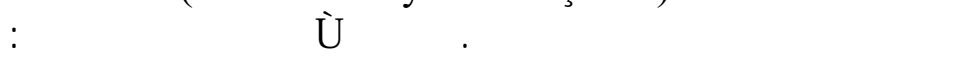

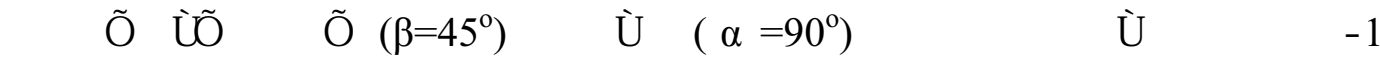

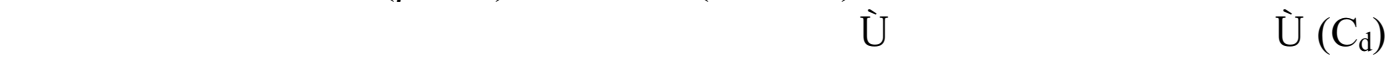

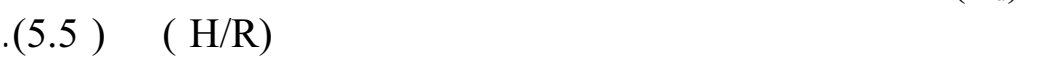

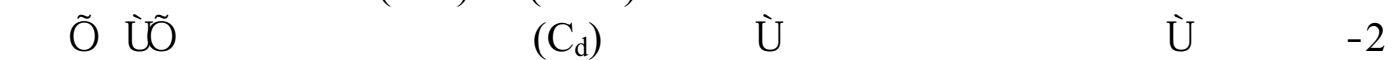

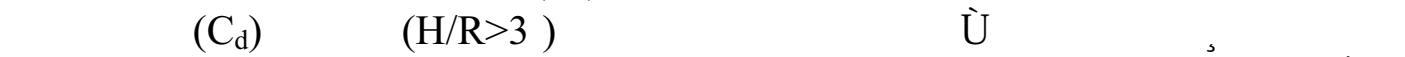

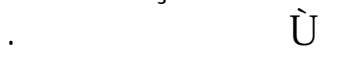

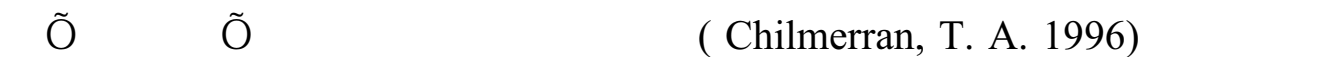

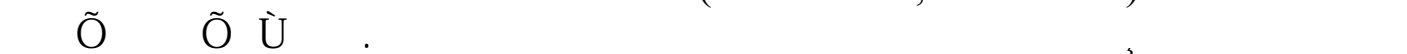

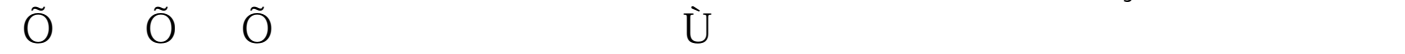

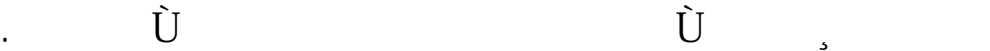

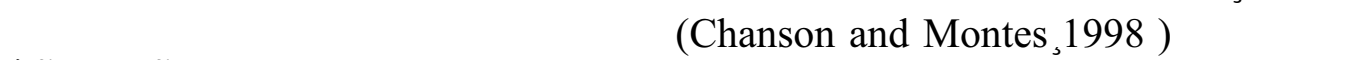

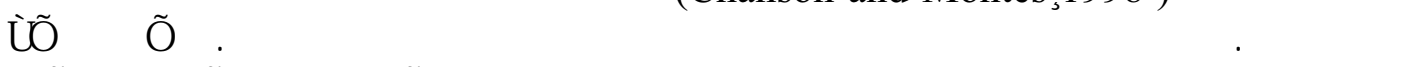

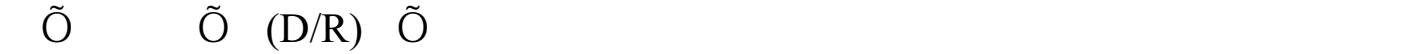

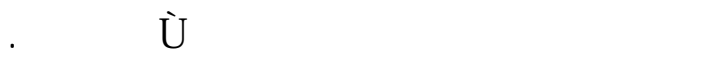

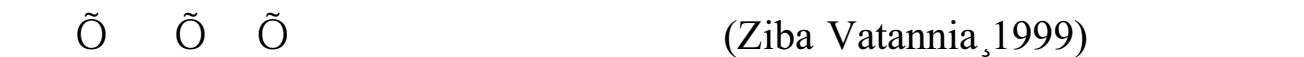

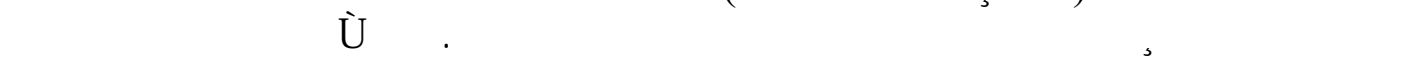

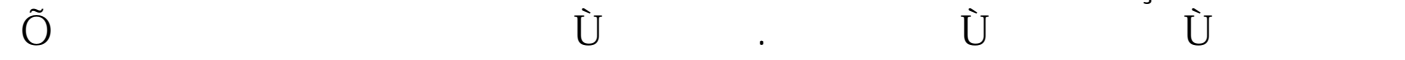

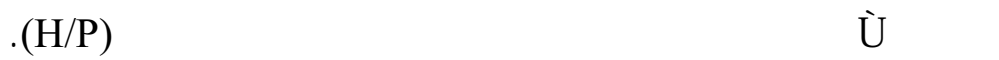

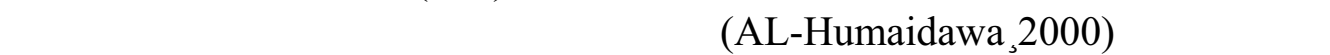

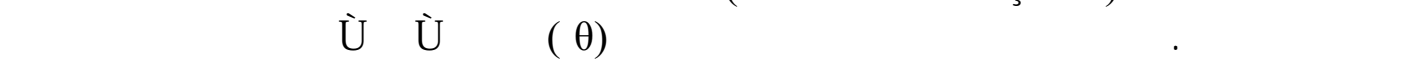

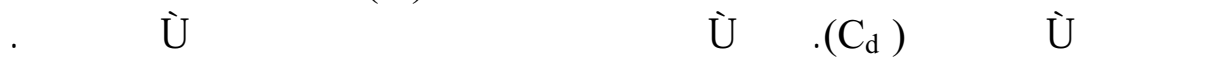

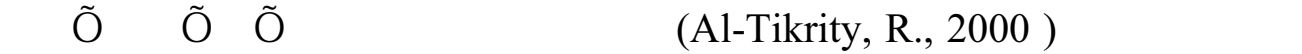

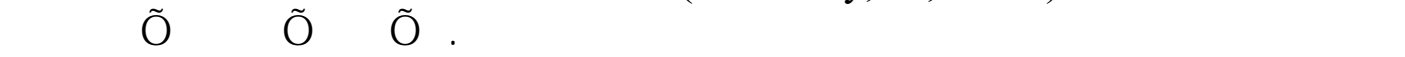

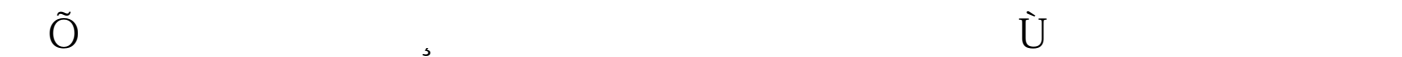

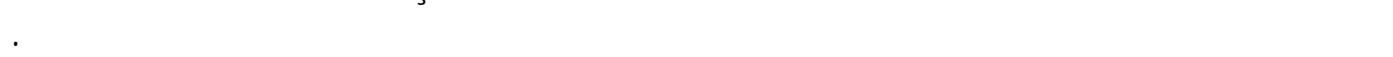

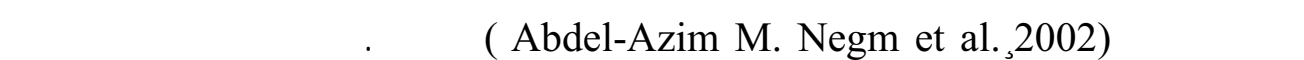

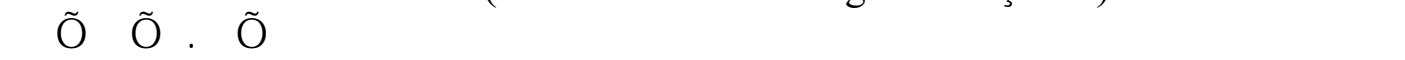

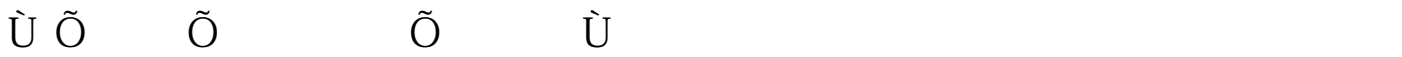

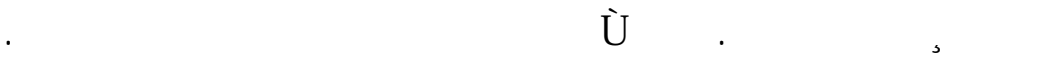

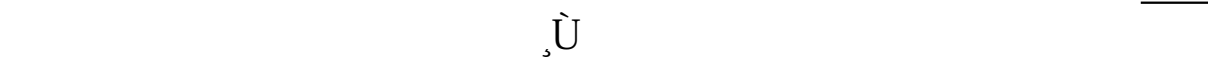

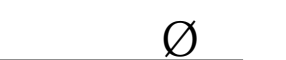
ry

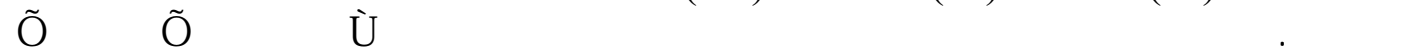

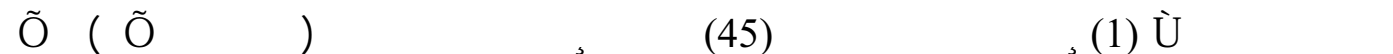

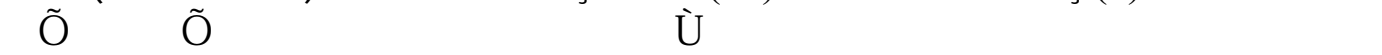

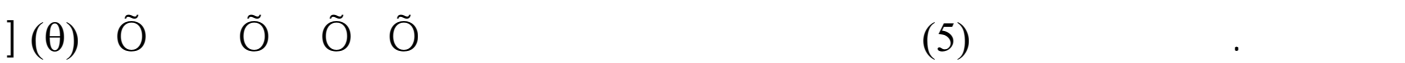

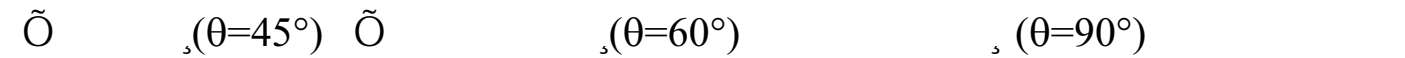




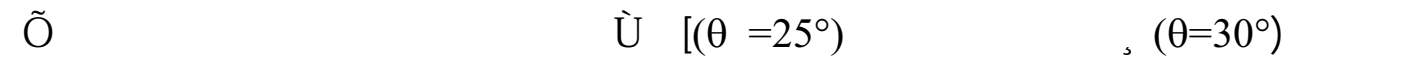
$\left(\beta=60^{\circ}\right)$ В ř

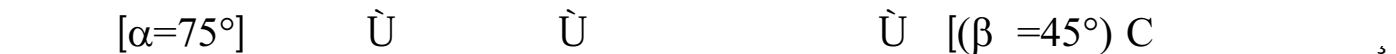

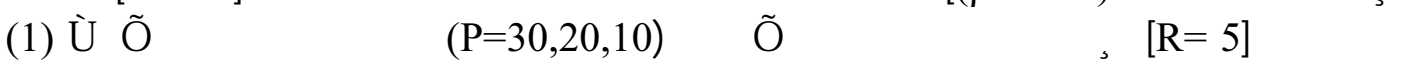

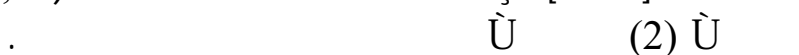
Śr̛̃

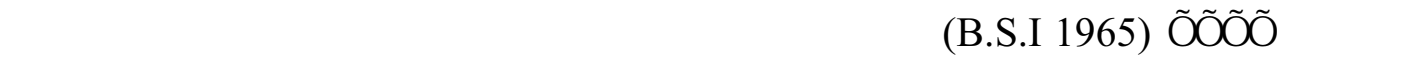

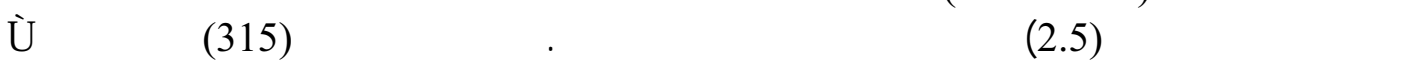

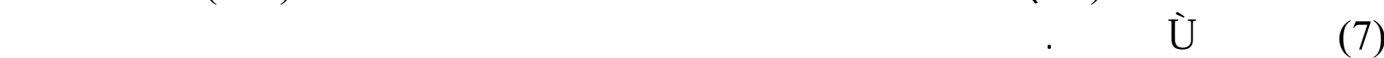

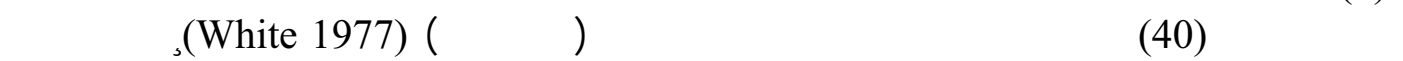

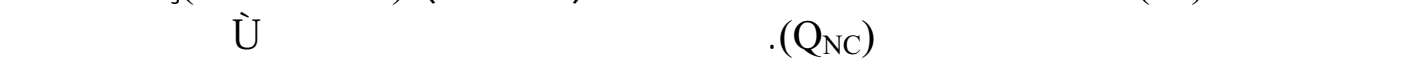

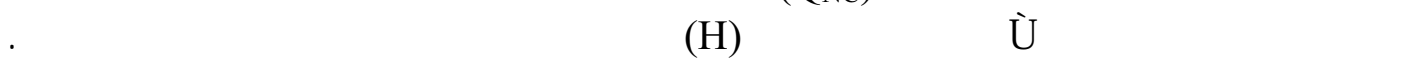

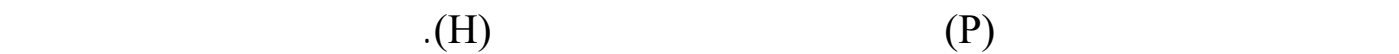

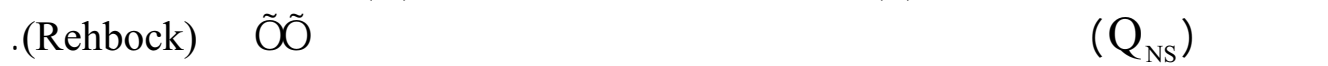

$\mathrm{Q}_{\mathrm{NS}}=\frac{2}{3} \mathrm{C}_{\mathrm{d}} \sqrt{2 \mathrm{~g}} \mathrm{~L} \mathrm{H}_{\mathrm{e}}{ }^{1.5}$

$\left.\mathrm{C}_{\mathrm{d}}=0.602+0.083 \frac{\mathrm{H}}{\mathrm{P}}\right)$

$\mathrm{H}_{\mathrm{e}}=(\mathrm{H}+0.0012)$

:Śf́s

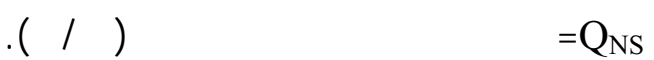

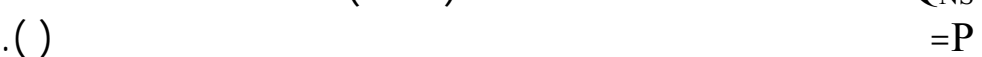

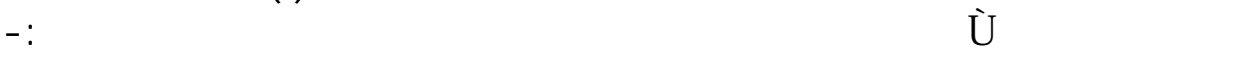

$\mathrm{C}_{\mathrm{d}}=\frac{Q_{N C}}{\frac{2}{3} \sqrt{2 g} L H^{1.5}}$

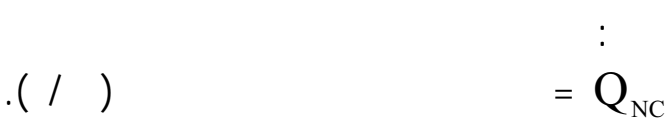

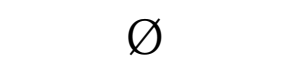

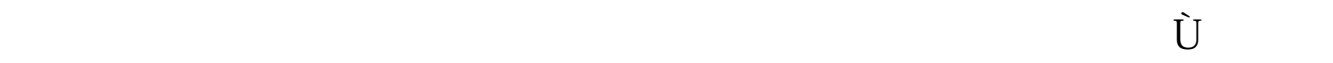

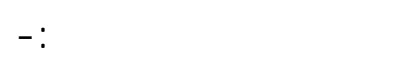

$\mathrm{f}(\mathrm{q}, \mathrm{H}, \mathrm{P}, \mathrm{g}, \rho, \mu, \beta, \theta)=0$

$: \hat{\not} \not$

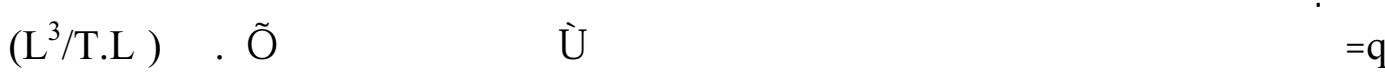

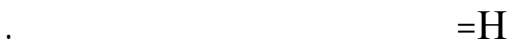

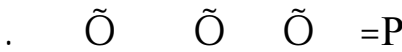
.Gบ ũt Ŕं 


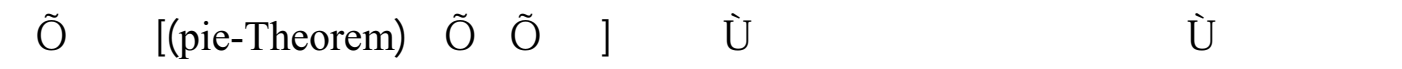

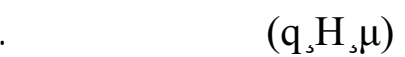

$\frac{\mathrm{Q}_{\mathrm{NC}}}{\frac{2}{3} * \sqrt{2 \mathrm{~g}} * \mathrm{~B} * \mathrm{H}^{3 / 2}}=\mathrm{C}_{\mathrm{d}}=\mathrm{f}_{1}\left(\frac{\mathrm{H}}{\mathrm{P}}, \mathrm{Re}, \beta, \theta\right)$

$\left.\mathrm{C}_{\mathrm{d}}=\mathrm{f}_{2}\left(\frac{\mathrm{H}}{\mathrm{P}}\right), \beta, \theta\right)$

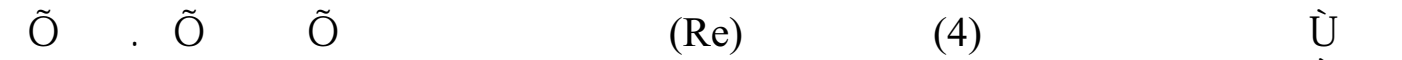

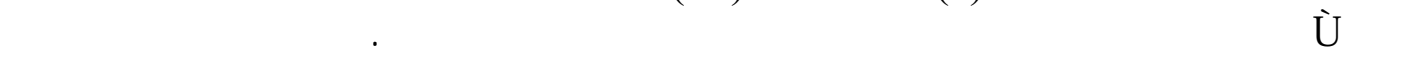

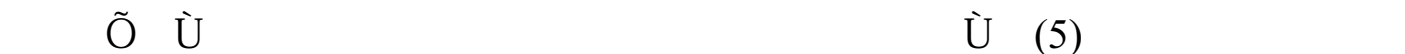

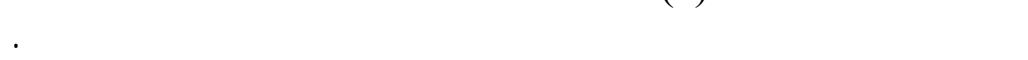

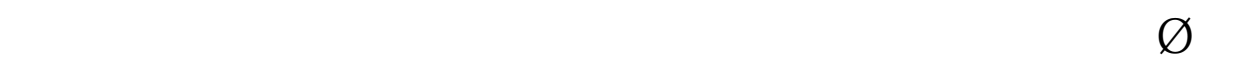

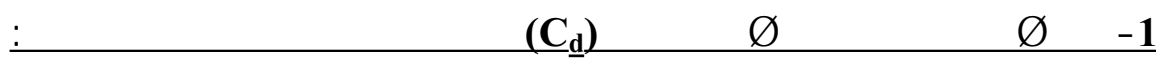

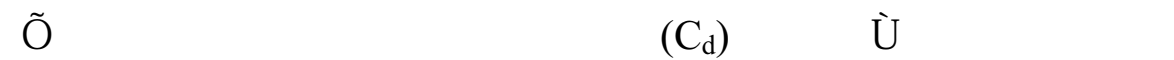

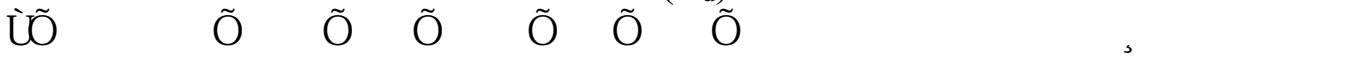

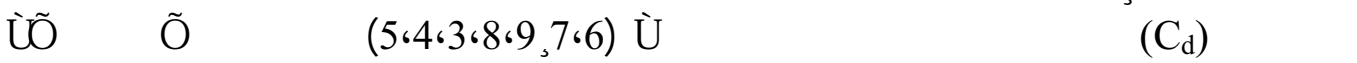

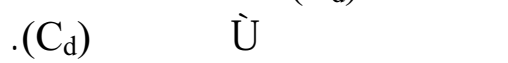

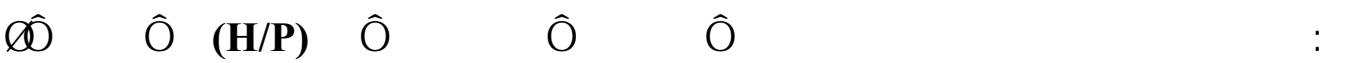

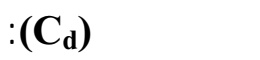

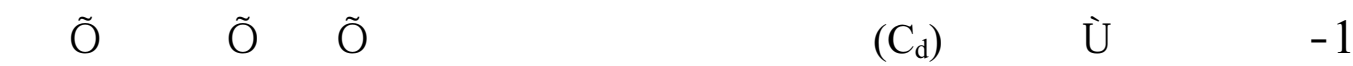

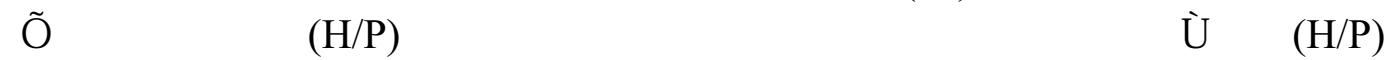

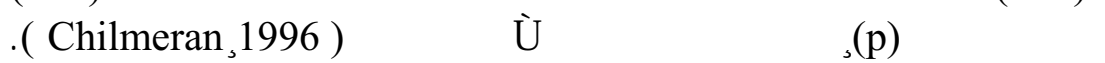

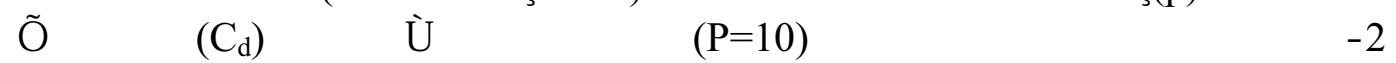

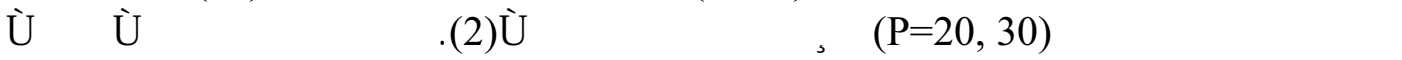

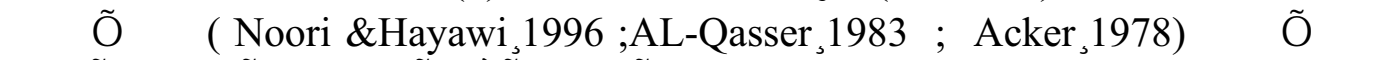

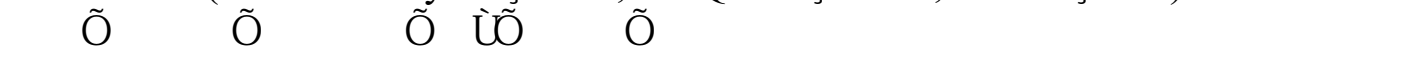

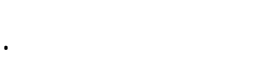

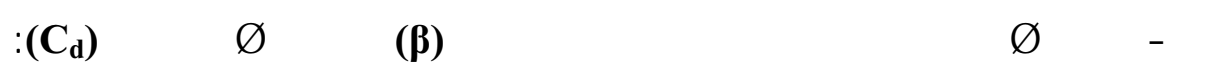

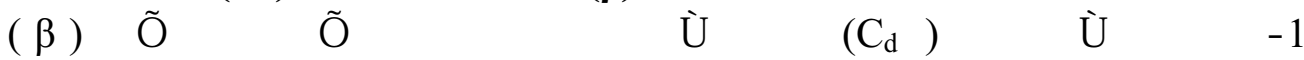

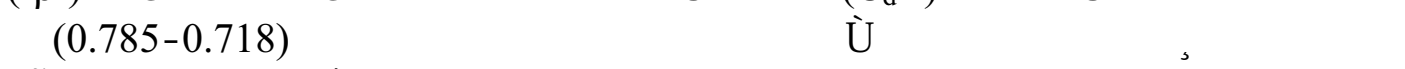

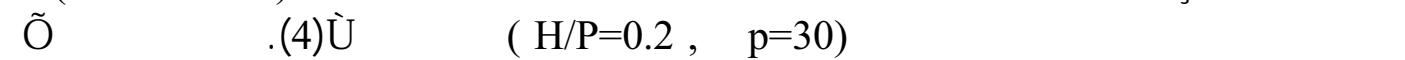

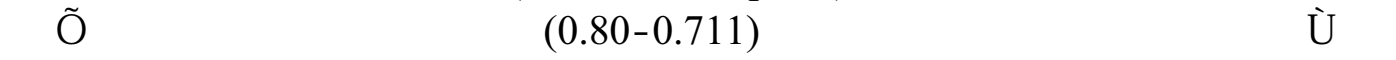

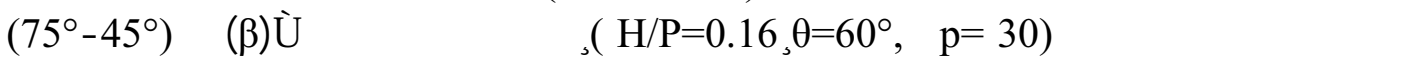




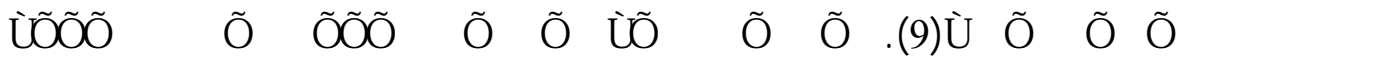

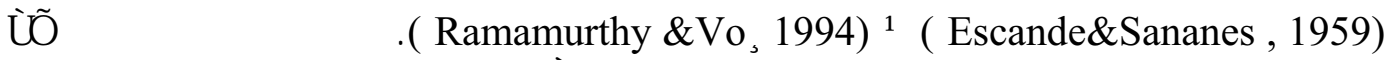

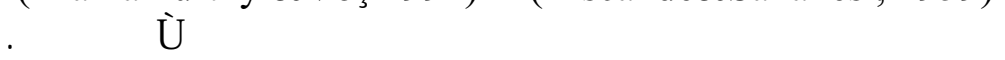

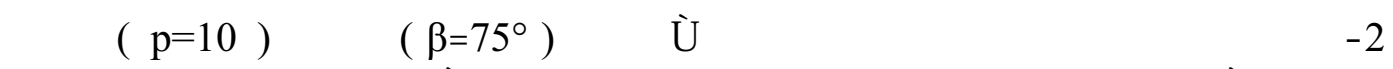

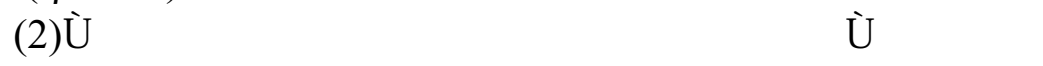

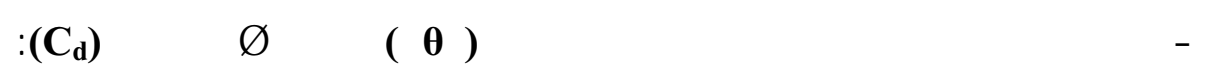

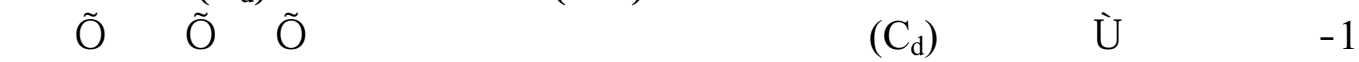

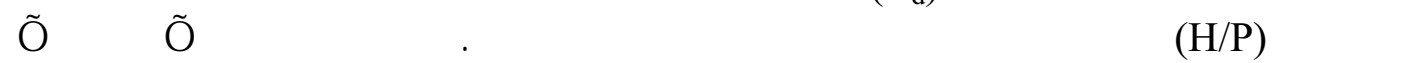

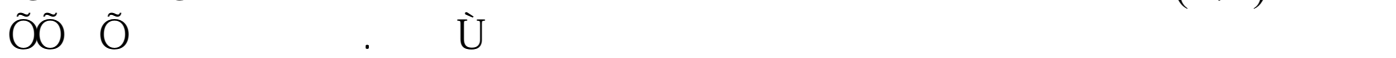

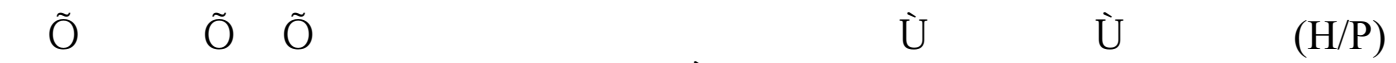

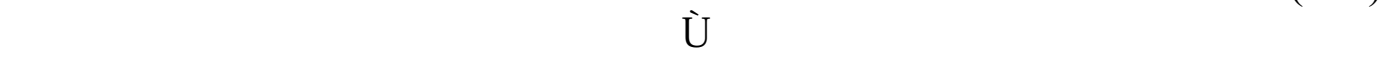

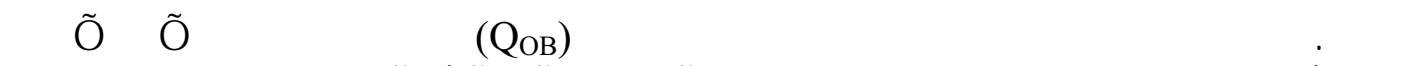

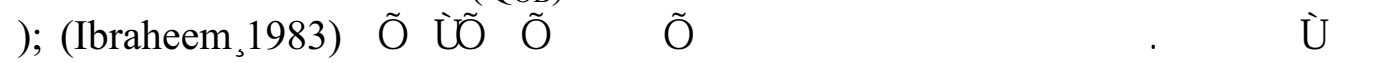
. (Noori \& Hayawi 999 ) ; (Noori \& HayawiD996) ; (Chilmeran口996

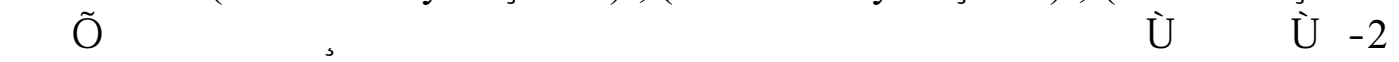

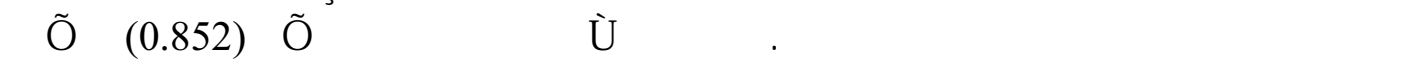

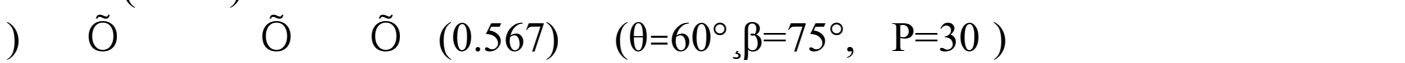

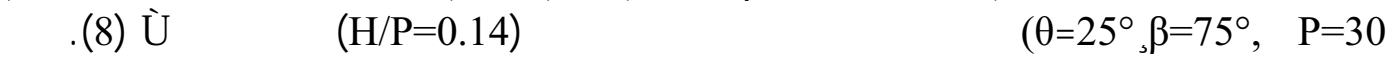

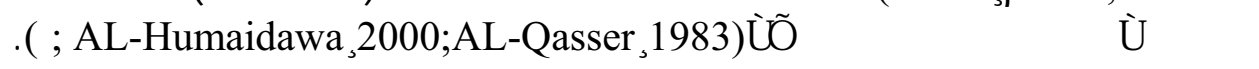

\section{:(C}

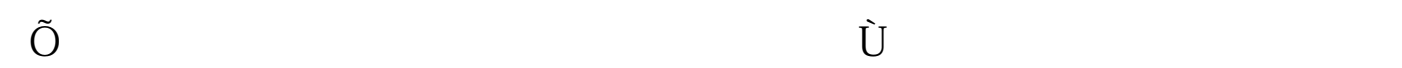

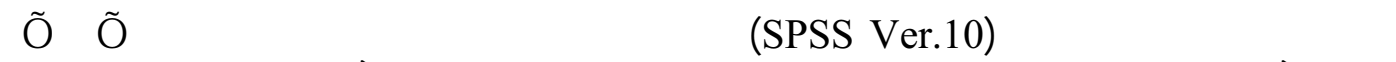

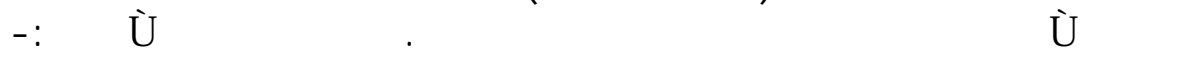

$\mathrm{C}_{\mathrm{d}}=0.7883 *\left[\left(\frac{\mathrm{H}}{\mathrm{P}}\right)^{0.01748} *(\operatorname{Sin} \theta)^{0.5131} *(\beta)^{0.3127}\right]$

:Ŝ甘

.(Degrees)Śř́š̃toứ $=\theta$

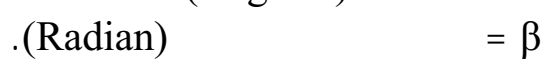

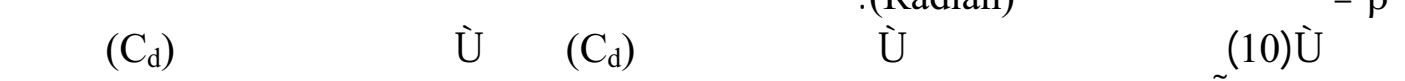
.

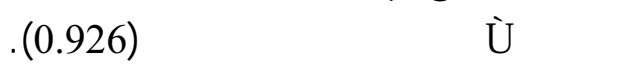

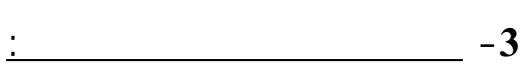

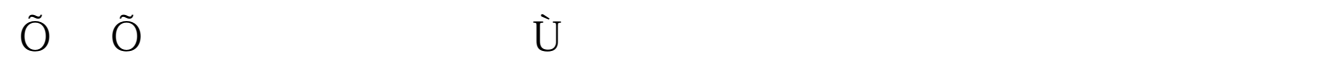

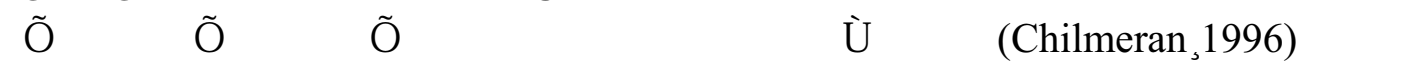

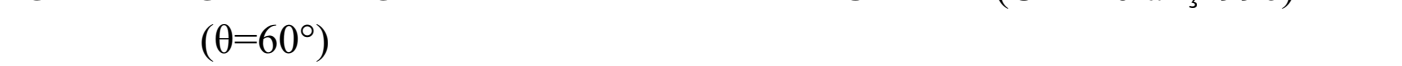

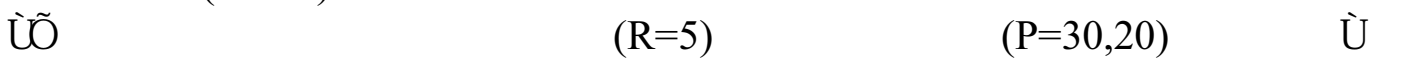
G) ŽĐ 


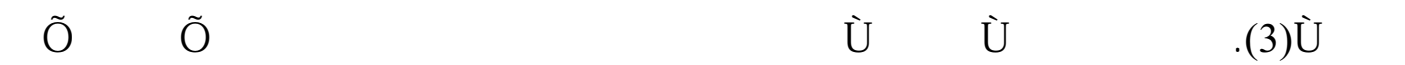

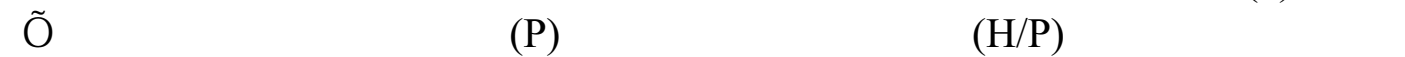

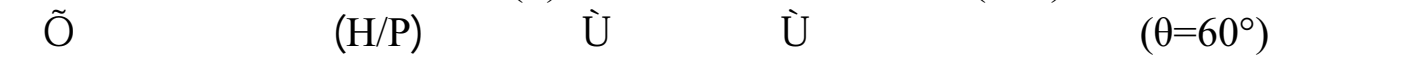

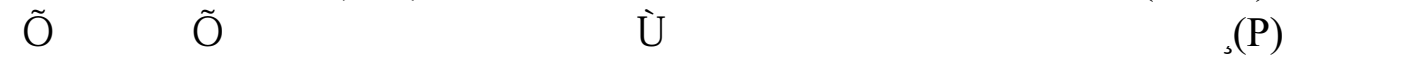

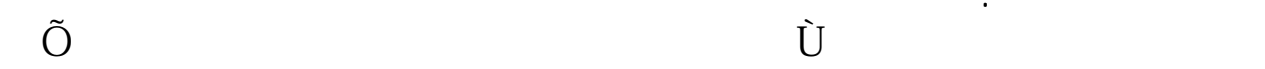

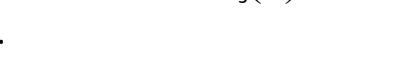

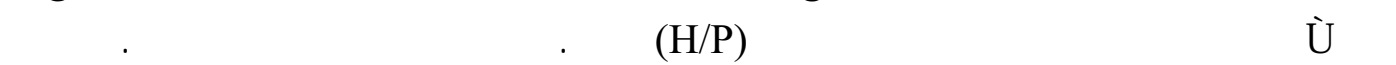
Escande \& ) Đ .(Ramamurthy \&Vo, 1994);(Sananes, 1959

ĐU్

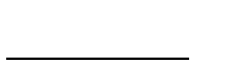

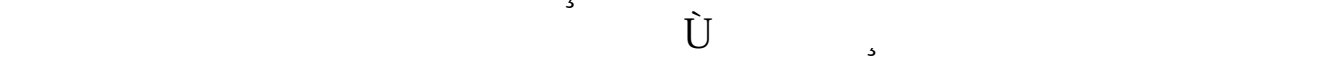
. Gsfứ

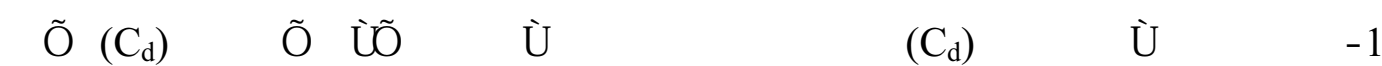

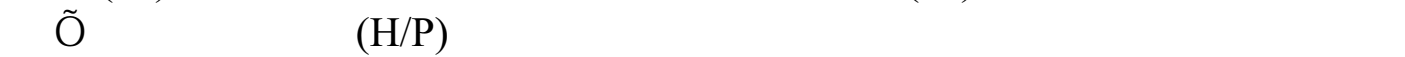

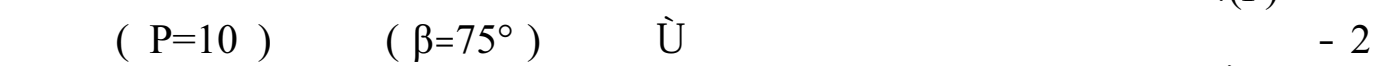

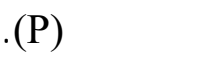

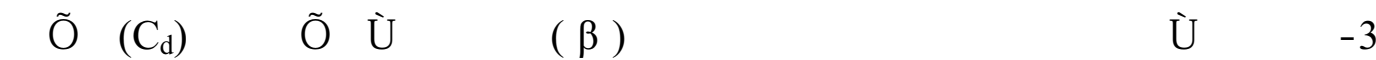

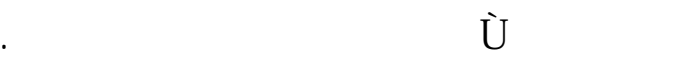

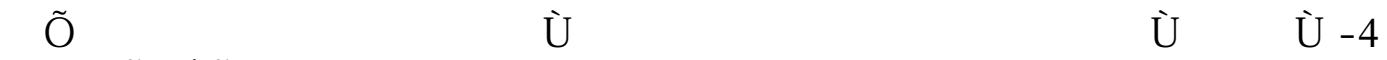

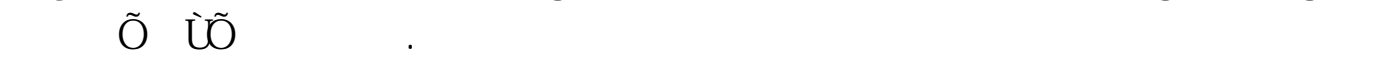

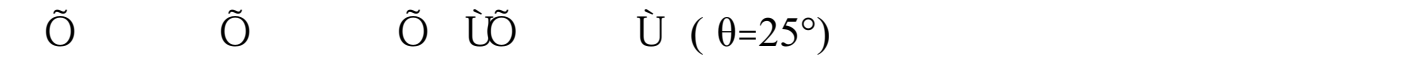
. $\left(\theta=60^{\circ}\left[45^{\circ}\left[\beta 0^{\circ}\right) \quad\right.\right.$ ũ

ttdions

1. Abdul - Azim' M. Negm, Al - Brahim' A. M. and Alhamid' A. A., (2002),"Combined free flow over weirs and below gates", Journal of Hydraulic Research. Vol. 40, No. 3.

2. Abed Ali, H. S.,(1989), "Characteristics of the flow over the roundcrested weirs", M. Sc. Thesis Dept. of Civil Engineering, University of Basra, Iraq.

3. Acker, P.' white, W., parkins, J. and Harrison, A.,(1978), "Weirs and flume for flow measurement", First edition, John Wiley and Sons Co., Chicheestter, England, U. K.

4. Al-A' rage, Ayman, T. H. A. (2001), "Experimental investigation on the hydraulic performance of duckbill weirs", M.Sc. thesis, Dept. of civil Eng., University of Al-Mustansiriyah, Iraq. 
5. Al-Humaidawe, Amer H., T., (2000), "Characteristics of flow over oblique broad crested weir", M. Sc., Thesis, University of AlMustansiriyah, Iraq.

6. Al-Qasser, G., (1983), "Laboratory investigation of some hydraulic features of Labyrinth weirs", M. Sc., Thesis, University of Baghdad, Iraq.

7. Al-Tikrity, Rokaia A., (2000), "Discharge coefficient for semicircular crested rectangular weirs", M.Sc., Thesis, Dep. of Civil Engineering, University of Tikrit, Iraq.

8. British Standard Institution (BSI). , (1965), "Method of measurement of liquid flow in open channel", part 4A, BS 3680, London, England, U. K.

9. Chanson, H., and Montes, J. S., (1998), "Over flow characteristics of circular weirs. Effects of inflow conditions", Journal of Irrigation and Drainage Engineering, Vol. 124, No. 3, May/June.

10. Chilmeran, T., (1996), "Characteristics of flow over normal and oblique weirs with semicircular crest", M. Sc., thesis, University of Mosul, Iraq.

11. Ramamurthy, S.Amruthur, and Diep Vo-Ngoc, (1994),"Characteristics of circular-crested weir", Journal of Hydraulic Engineering, Vol.119.No.9,September.

12. Ibraheem, G., (1988), "Discharge and flow characteristics of weirs with triangular plan form”, M. Sc. thesis, University of Mosul. Iraq.

13. Noori, B. and Hayawi, G., (1996), "Hydraulic characteristics of flow over semicircular sharp-crested weirs", Journal of dirasat for engineering science, Jordan vol.23, No. 1, pp (37-46).

14. Noori, B. and Hayawi, H., (1999), "Hydraulic characteristics of flow over sharp-crested zigzag weirs ", Journal of dirasat for engineering sciences, Jordan vol. 26, No.1, pp (135-146).

15. Ramamurthy, S. Amruthur, and Diep VO - Ngoc, (1994), "Characteristics of circular - crested weir", Journal of Hydraulic Engineering, Vol. 119. No. 9, September 8.

16. White, W. R., (1977), “Thin plate weirs”, Proc. Inst. Civil engineering, part.2, 63 .

17. Ziba, V., (1999), "Discharge coefficient of oblique sharp crested weirs using experimental results", M. Sc. Thesis, Dept. of Civil Engineering, Sharif University of Technology, Tehran, Iran. (Abstract). 


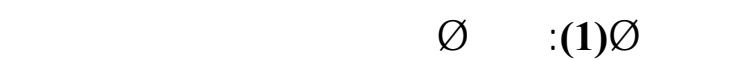

\begin{tabular}{|c|c|c|c|c|c|c|c|c|}
\hline $\begin{array}{l}\text { Crest Height } \\
\text { P(cm) }\end{array}$ & $\begin{array}{l}\text { Crest } \\
\text { Length } \\
\text { L (cm) }\end{array}$ & $\begin{array}{c}\text { Downstream } \\
\text { angle ( } \beta \text { ) }\end{array}$ & $\begin{array}{l}\text { Upstream } \\
\text { angle }(\alpha)\end{array}$ & $\begin{array}{c}\text { Series } \\
\text { No. }\end{array}$ & $\begin{array}{c}\text { Crest } \\
\text { Radius } \\
\mathrm{R}(\mathrm{cm})\end{array}$ & $\begin{array}{l}\text { ObLique } \\
\text { angle ( } \theta)\end{array}$ & $\begin{array}{c}\text { ModeL } \\
\text { No. }\end{array}$ & $\begin{array}{c}\text { group } \\
\text { No. }\end{array}$ \\
\hline $30,20,10$ & 30 & $75^{\circ}$ & \multirow{3}{*}{$75^{\circ}$} & $\mathbf{A}$ & \multirow{3}{*}{5} & \multirow{3}{*}{$\begin{array}{c}90^{\circ} \\
\text { (Normal } \\
\text { weir) }\end{array}$} & $1-3$ & \multirow{3}{*}{1} \\
\hline $30,20,10$ & 30 & $60^{\circ}$ & & B & & & $4-6$ & \\
\hline $30,20,10$ & 30 & $45^{\circ}$ & & $\mathrm{C}$ & & & $7-9$ & \\
\hline $30,20,10$ & 34.6 & $75^{\circ}$ & \multirow{3}{*}{$75^{\circ}$} & $\mathbf{A}$ & \multirow{3}{*}{5} & \multirow{3}{*}{$60^{\circ}$} & $10-12$ & \multirow{3}{*}{2} \\
\hline $30,20,10$ & 34.6 & $60^{\circ}$ & & B & & & $13-15$ & \\
\hline $30,20,10$ & 34.6 & $45^{\circ}$ & & $\mathrm{C}$ & & & $16-18$ & \\
\hline $30,20,10$ & 42.424 & $75^{\circ}$ & \multirow{3}{*}{$75^{\circ}$} & $\mathbf{A}$ & \multirow{3}{*}{5} & \multirow{3}{*}{$45^{\circ}$} & $19-21$ & \multirow{3}{*}{3} \\
\hline $30,20,10$ & 42.424 & $60^{\circ}$ & & B & & & $22-24$ & \\
\hline $30,20,10$ & 42.424 & $45^{\circ}$ & & $\mathrm{C}$ & & & $23-27$ & \\
\hline $30,20,10$ & 60 & $75^{\circ}$ & \multirow{3}{*}{$75^{\circ}$} & $\mathbf{A}$ & \multirow{3}{*}{5} & \multirow{3}{*}{$30^{\circ}$} & $28-30$ & \multirow{3}{*}{4} \\
\hline $30,20,10$ & 60 & $60^{\circ}$ & & B & & & $31-33$ & \\
\hline $30,20,10$ & 60 & $45^{\circ}$ & & C & & & $34-36$ & \\
\hline $30,20,10$ & \begin{tabular}{|l|}
70.98 \\
\end{tabular} & $75^{\circ}$ & \multirow{3}{*}{$75^{\circ}$} & $\mathbf{A}$ & \multirow{3}{*}{5} & \multirow{3}{*}{$25^{\circ}$} & $37-39$ & \multirow{3}{*}{5} \\
\hline $30,20,10$ & 70.98 & $60^{\circ}$ & & B & & & $40-42$ & \\
\hline $30,20,10$ & \begin{tabular}{|l|}
70.98 \\
\end{tabular} & $45^{\circ}$ & & C & & & $43-45$ & \\
\hline
\end{tabular}

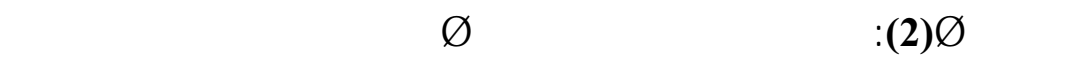

\begin{tabular}{|c|c|c|c|c|c|c|c|c|c|c|}
\hline \multicolumn{9}{|c|}{ 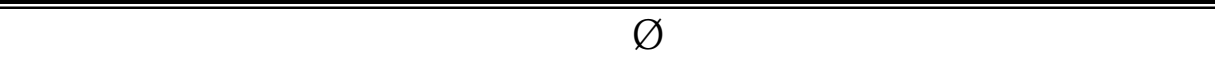 } & \multirow{3}{*}{$\left(\theta^{\circ}\right)$} & \multirow{3}{*}{$\begin{array}{l}\mathrm{P} \\
\text { (c } \\
\mathrm{m})\end{array}$} \\
\hline \multicolumn{3}{|c|}{$\beta=45^{\circ}$} & \multicolumn{3}{|c|}{$\beta=60^{\circ}$} & \multicolumn{3}{|c|}{$\beta=75^{\circ}$} & & \\
\hline Min. & Max. & $\mathrm{H} / \mathrm{P}$ & Min. & Max. & $\mathrm{H} / \mathrm{P}$ & Min. & Max. & $\mathrm{H} / \mathrm{P}$ & & \\
\hline 0.750 & 0.821 & 0.860 & 0.777 & 0.856 & 0.835 & 0.838 & 0.899 & 0.810 & ${ }^{\circ} 90$ & \multirow{5}{*}{10} \\
\hline 0.643 & 0.743 & 0.530 & 0.687 & 0.818 & 0.480 & 0.750 & 0.931 & 0.402 & $60^{\circ}$ & \\
\hline 0.586 & 0.675 & 0.440 & 0.635 & 0.743 & 0.400 & 0.692 & 0.847 & 0.320 & ${ }^{\circ} 45$ & \\
\hline 0.466 & 0.553 & 0.405 & 0.514 & 0.649 & 0.300 & 0.584 & 0.671 & 0.340 & ${ }^{\circ} 30$ & \\
\hline 0.453 & 0.567 & 0.300 & 0.473 & 0.586 & 0.300 & 0.516 & 0.632 & 0.310 & ${ }^{\circ} 25$ & \\
\hline 0.711 & 0.758 & 0.400 & 0.747 & 0.809 & 0.404 & 0.790 & 0.850 & 0.395 & ${ }^{\circ} 90$ & \multirow{5}{*}{20} \\
\hline 0.599 & 0.711 & 0.265 & 0.639 & 0.782 & 0.230 & 0.709 & 0.871 & 0.210 & $60^{\circ}$ & \\
\hline 0.554 & 0.651 & 0.210 & 0.602 & 0.704 & 0.218 & 0.649 & 0.795 & 0.215 & ${ }^{\circ} 45$ & \\
\hline 0.438 & 0.522 & 0.205 & 0.485 & 0.617 & 0.155 & 0.556 & 0.649 & 0.150 & $30^{\circ}$ & \\
\hline 0.424 & 0.530 & 0.150 & 0.447 & 0.558 & 0.155 & 0.486 & 0.584 & 0.170 & $25^{\circ}$ & \\
\hline 0.664 & 0.743 & 0.233 & 0.709 & 0.790 & 0.240 & 0.73 & 0.825 & 0.237 & $90^{\circ}$ & \multirow{5}{*}{30} \\
\hline 0.586 & 0.693 & 0.160 & 0.619 & 0.759 & 0.155 & 0.675 & 0.852 & 0.137 & $60^{\circ}$ & \\
\hline 0.531 & 0.636 & 0.145 & 0.588 & 0.689 & 0.145 & 0.637 & 0.785 & 0.103 & $45^{\circ}$ & \\
\hline 0.427 & 0.507 & 0.143 & 0.474 & 0.600 & 0.123 & 0.536 & 0.639 & 0.103 & $30^{\circ}$ & \\
\hline 0.412 & 0.498 & 0.107 & 0.429 & 0.548 & 0.100 & 0.475 & 0.567 & 0.127 & $25^{\circ}$ & \\
\hline
\end{tabular}




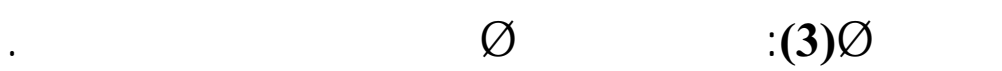

\begin{tabular}{|c|c|c|c|c|c|}
\hline \multirow{2}{*}{$(2-1)$} & $\overline{(2)}$ & (1) & \multirow[b]{3}{*}{$\mathbf{H} / \mathbf{P}$} & \multirow{3}{*}{ 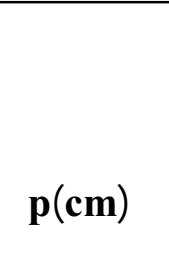 } & \multirow{3}{*}{ 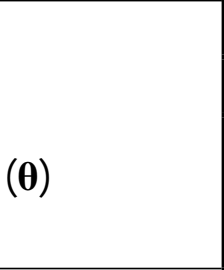 } \\
\hline & $\left(\beta=90^{\circ}\right)$ & $\left(\beta=75^{\circ}\right)$ & & & \\
\hline$\Delta \mathrm{Cd}(\%)$ & 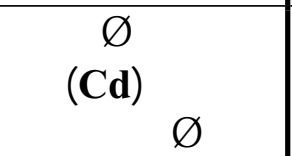 & 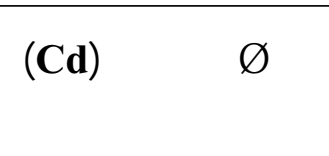 & & & \\
\hline-24.11 & 0.591 & 0.7335 & 0.14 & \multirow{5}{*}{30} & \multirow{10}{*}{$90^{\circ}$} \\
\hline $\begin{array}{l}-7.63 \\
\end{array}$ & 0.695 & 0.748 & 0.16 & & \\
\hline-27.71 & 0.599 & 0.765 & 0.18 & & \\
\hline-29.00 & 0.607 & 0.783 & 0.2 & & \\
\hline-30.47 & 0.617 & 0.805 & 0.22 & & \\
\hline-35.04 & 0.585 & 0.790 & 0.28 & \multirow{5}{*}{20} & \\
\hline-36.22 & 0.588 & 0.801 & 0.3 & & \\
\hline-37.16 & 0.592 & 0.812 & 0.32 & & \\
\hline-38.32 & 0.595 & 0.823 & 0.34 & & \\
\hline-39.30 & 0.598 & 0.833 & 0.36 & & \\
\hline -19.01 & 0.710 & 0.845 & (20.14 & \multirow{5}{*}{30} & \multirow{10}{*}{$60^{\circ}$} \\
\hline-11.42 & 0.718 & 0.800 & 0.16 & & \\
\hline-6.59 & 0.713 & 0.760 & 0.18 & & \\
\hline-1.41 & 0.71 & 0.720 & 0.2 & & \\
\hline 0.00 & 0.706 & 0.706 & 0.22 & & \\
\hline-24.66 & 0.734 & 0.915 & 0.18 & \multirow{5}{*}{20} & \\
\hline-20.90 & 0.732 & 0.885 & 0.20 & & \\
\hline-17.65 & 0.731 & 0.860 & 0.22 & & \\
\hline-14.35 & 0.7311 & 0.836 & 0.24 & & \\
\hline-10.58 & 0.7325 & 0.810 & 0.26 & & \\
\hline
\end{tabular}

Rtüfūjọšsg

Fthotrione

ũ ướț̄ 

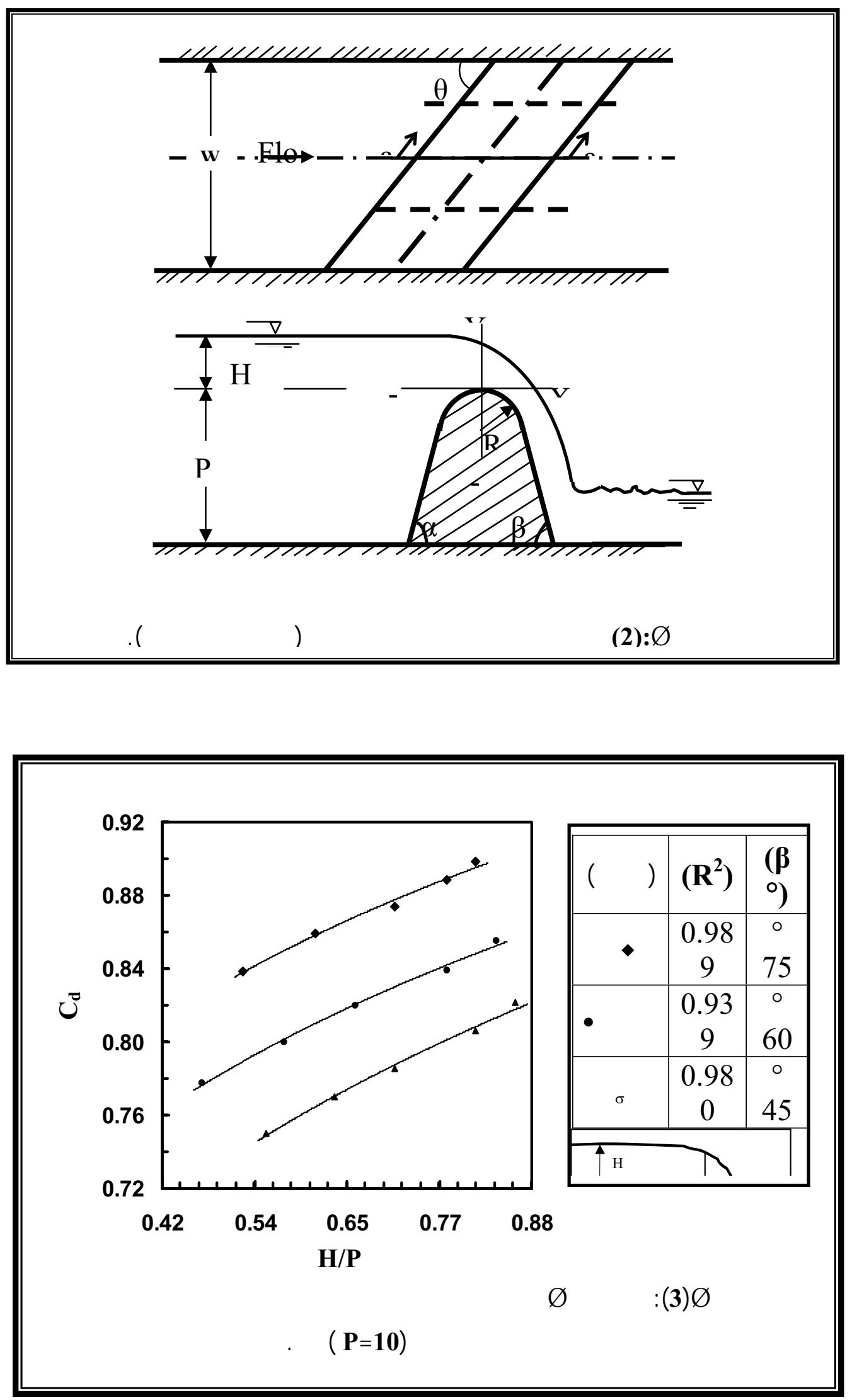


\begin{tabular}{llll} 
Al-Rafidain Engineering & Vol.14 & No.2 & 2006 \\
\hline
\end{tabular}
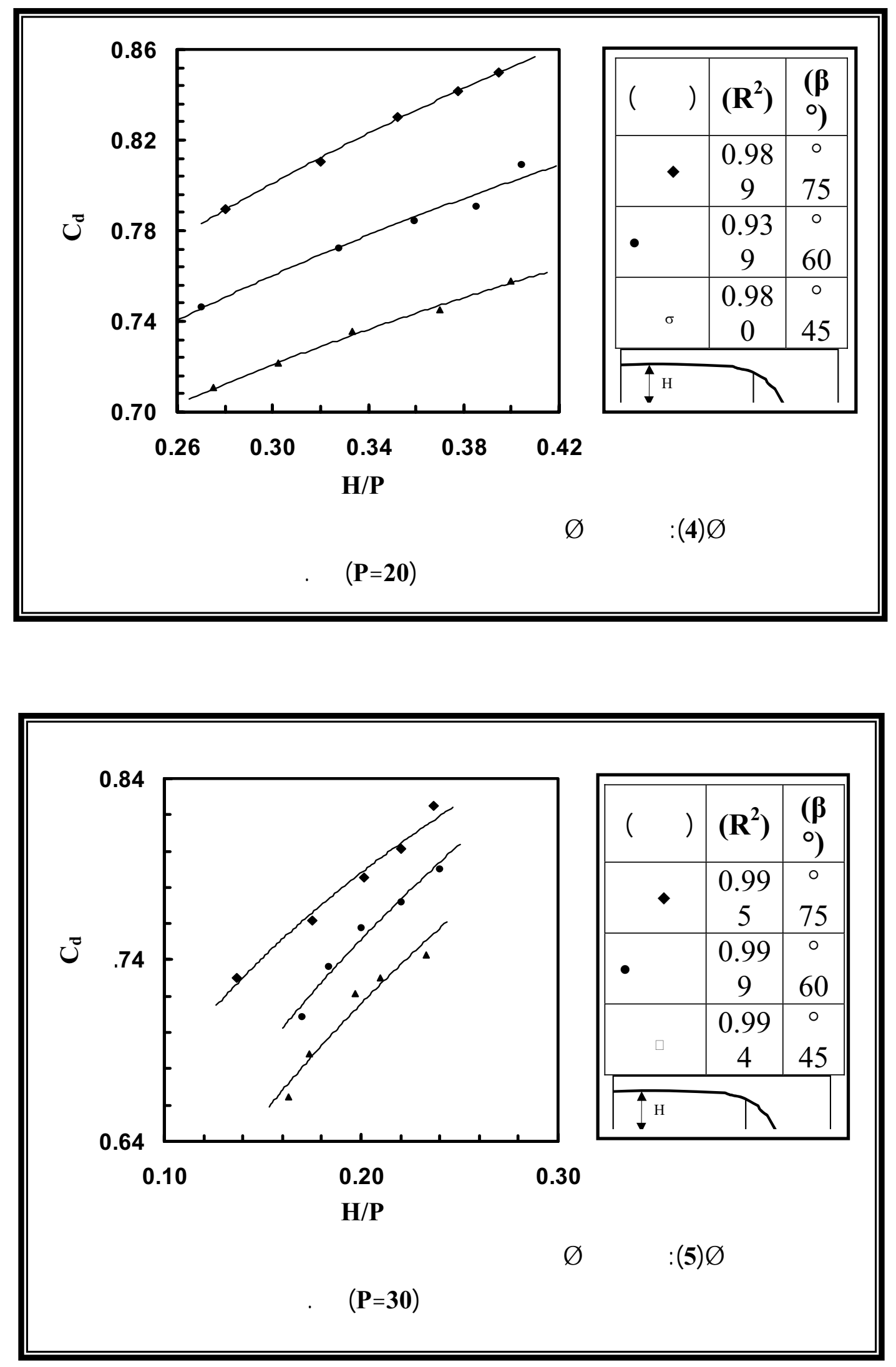

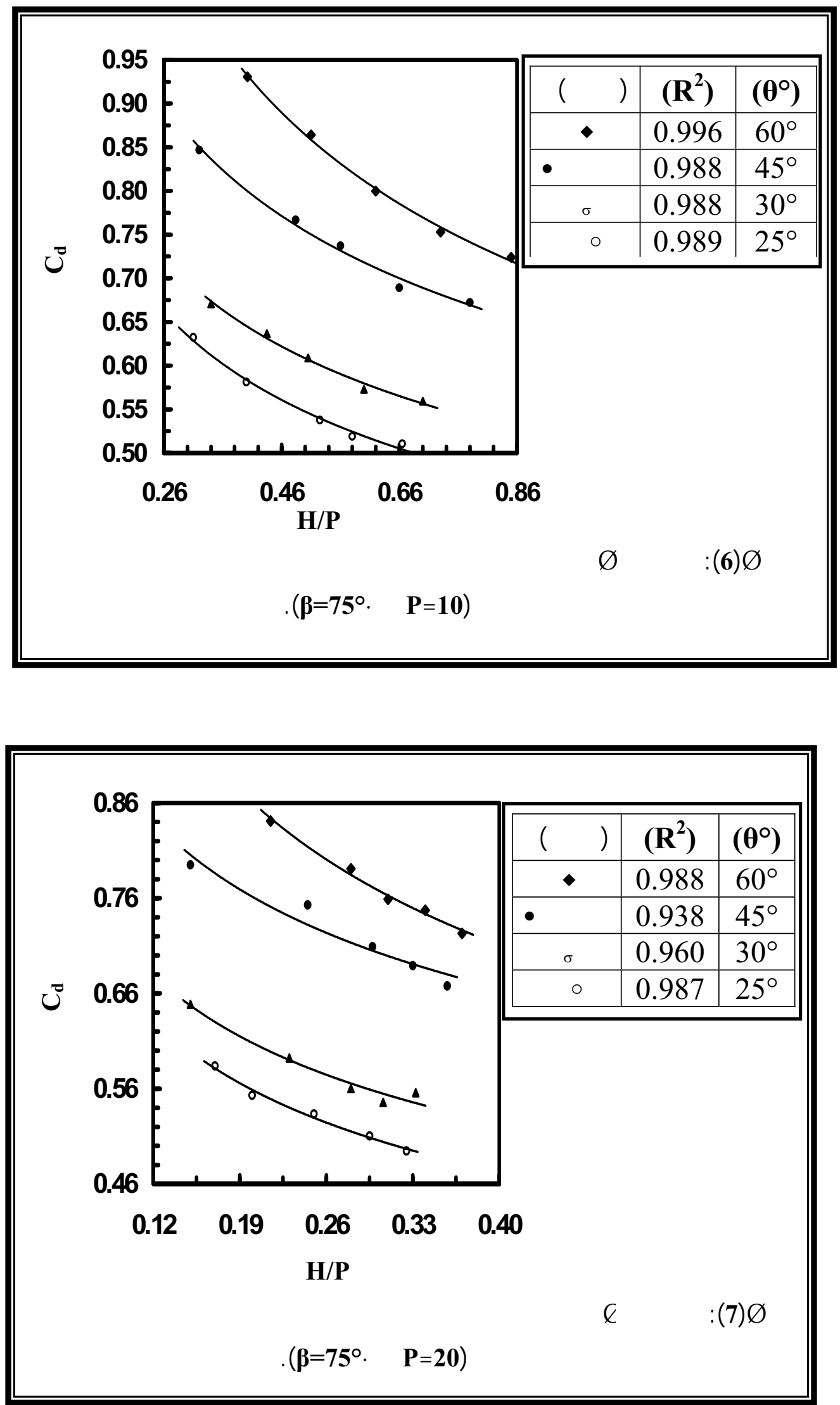


\begin{tabular}{llll} 
Al-Rafidain Engineering & Vol.14 & No.2 & 2006 \\
\hline
\end{tabular}

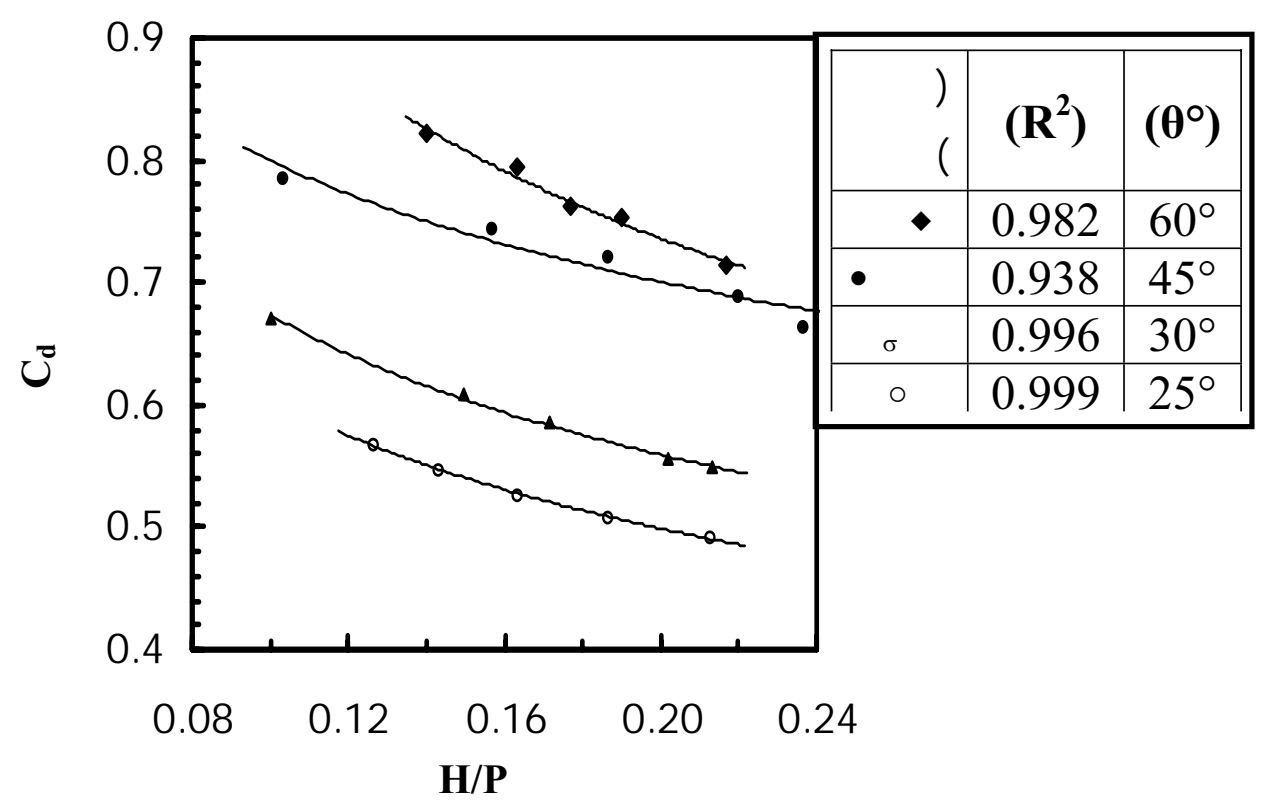

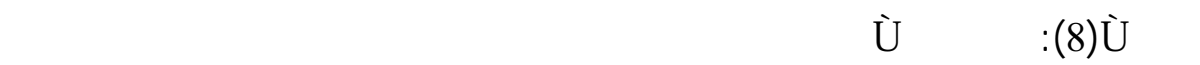
. $\left(\beta=75^{\circ}\left[{ }^{r} \mathrm{P}=30\right)\right.$ ũ

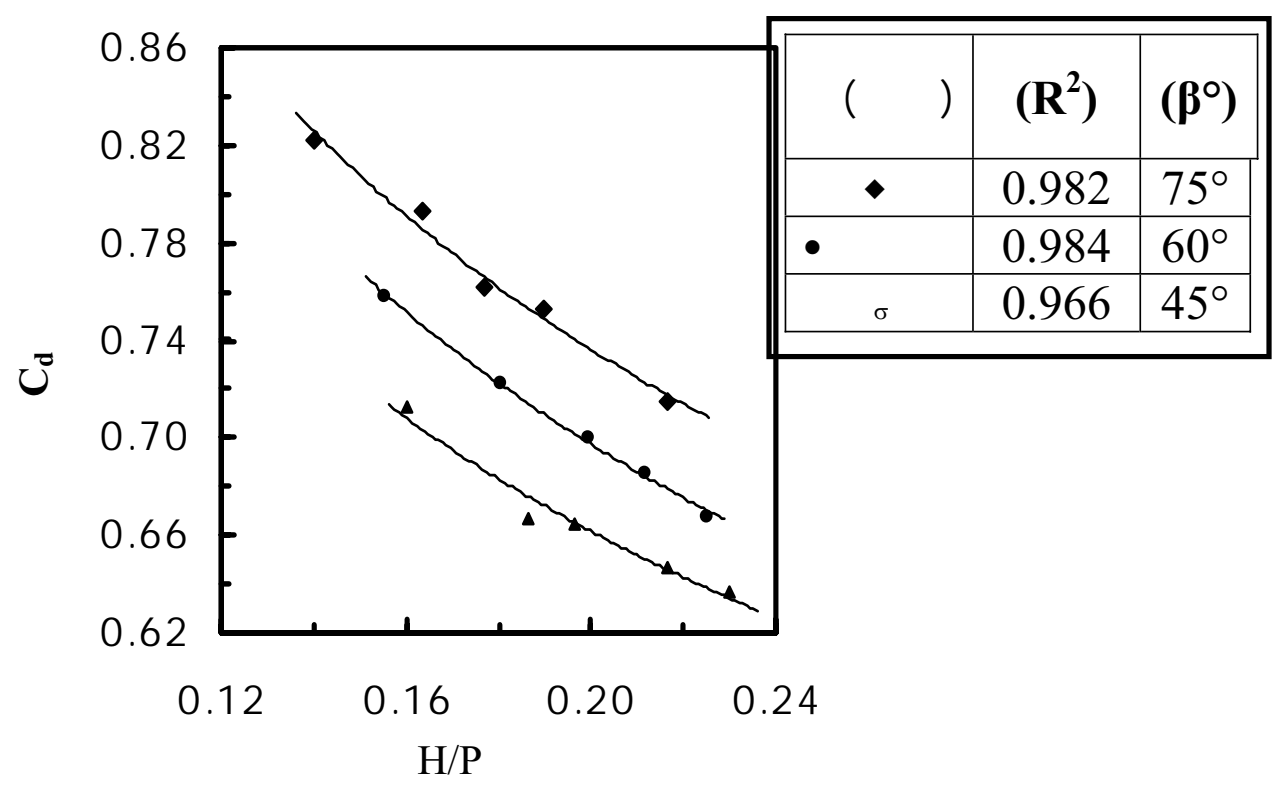

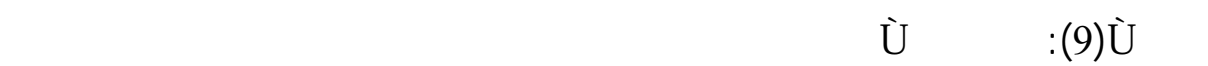

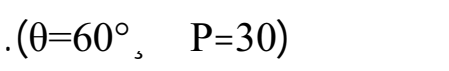




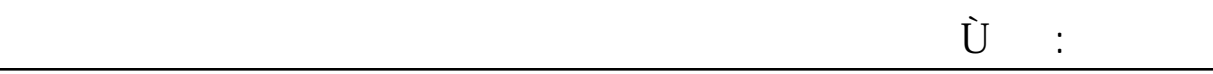

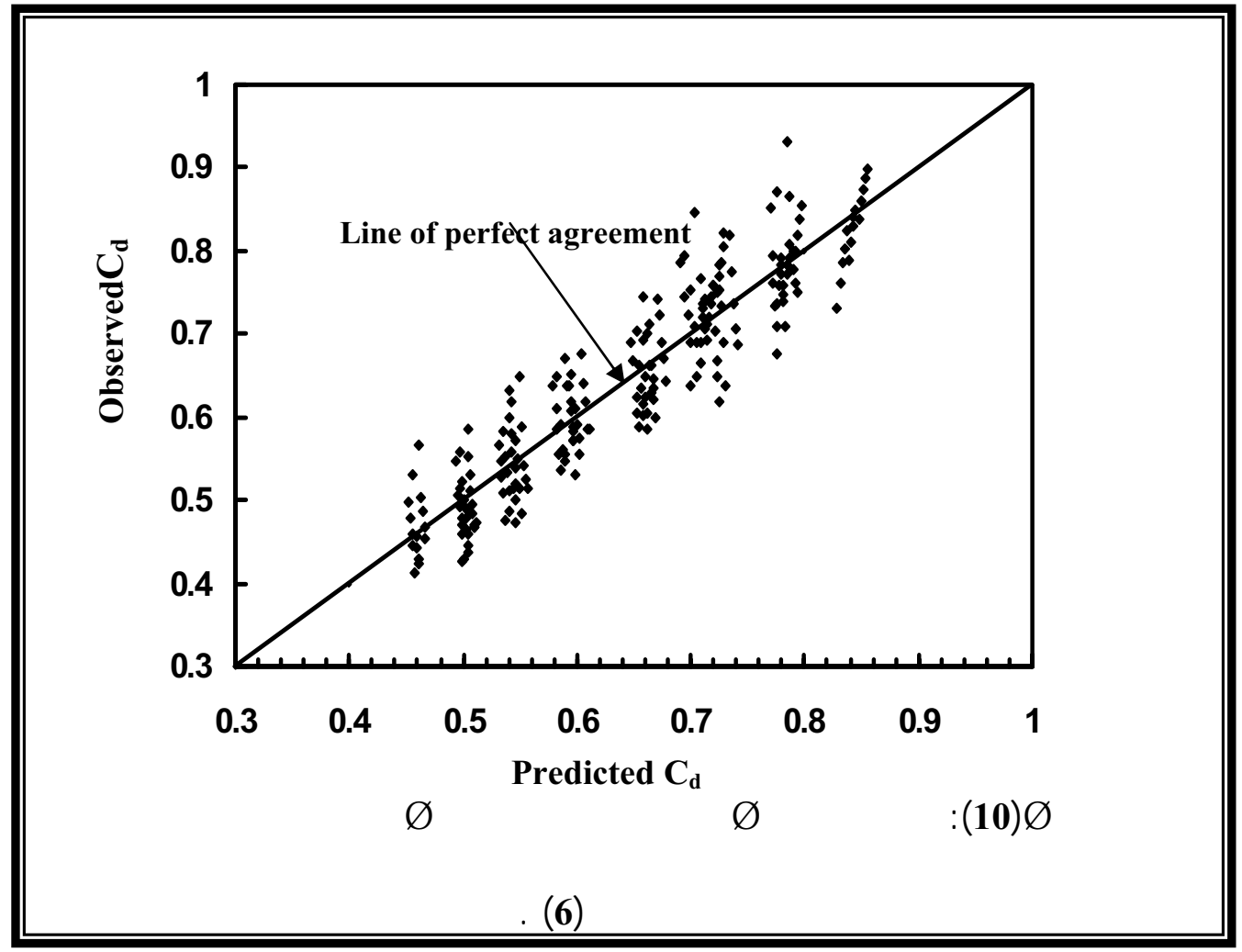

